ElisabeTH MeLon VIERA ${ }^{1}$

ManOEl ANTÔNIO dos SANTOS 2

Daniela Barsotti Santos

Marina Pasquali Marconato Mancin ${ }^{3}$ Hayala Cristina Cavenague de Souza ${ }^{4}$

JORGE LUIS BAZAN ${ }^{5}$

Gielci da Silva Castro Perdoná

\title{
Validação do Body Image Relationship Scale para mulheres com câncer de mama
}

\author{
Validation of Body Image Relationship Scale \\ for women with breast cancer
}

Artigo Original

Palavras-chave

Neoplasias da mama Imagem corporal Reprodutibilidade dos testes Estudos de validação

Keywords

Breast neoplasms

Body image

Reproducibility of results

Validation studies

Correspondênci

Flisabeth Meloni Vieir

Departamento de Medicina Social, Faculdade de Medicina de Ribeirão

Preto, Universidade de São Paulo

Avenida Bandeirantes, 3900 - Monte Alegre

CEP: $14049-900$

Ribeirão Preto (SP), Brasi

Recebido

13/04/2015

Aceito com modificacōes

$27 / 07 / 2015$

\section{Resumo}

OBJETIVO: Validar o instrumento Body Image Relationship Scale (BIRS) em mulheres brasileiras acometidas pelo câncer de mama. MÉTODOS: $\bigcirc$ instrumento foi aplicado por entrevistadoras treinadas em 139 usuárias do Sistema Único de Saúde que foram submetidas aos tratamentos do câncer entre 2006 e 2010 . O instrumento foi aferido considerandose a consistência interna e a confiabilidade. Para efeito de comparação as técnicas de análise fatorial utilizadas no artigo original foram aplicadas. RESULTADOS: $O$ valor de correlação Spearman-Brown foi 0,8, o que indica alto nível de confiabilidade, e o alfa de Cronbach encontrado foi 0,9 , indicando alto nível de consistência interna. A análise fatorial mostrou que quatro questões não tinham poder discriminatório e carga fatorial baixa e outras cinco foram realocadas em outros domínios. Dessa forma, foi aplicada e mostrou variabilidade semelhante ao instrumento original. CONCLUSÃO: A versão brasileira do BIRS, renomeada como Escala de Relacionamento e Imagem Corporal (ERIC), apresentou evidências de adequada confiabilidade e consistência interna, o que torna esse instrumento recomendável para aplicação em mulheres brasileiras com câncer de mama, apesar de alguns poucos limites.

\section{Abstract}

PURPOSE: To validate the instrument Body Image Relationship Scale (BIRS) for Brazilian women with breast cancer. METHODS: The instrument was administered by trained interviewers to 139 women who used the Brazilian Unified Health System (SUS). All of them had been submitted to cancer treatments between 2006 and 2010. The instrument was validated considering internal consistency and reliability. In order to compare the techniques, the same factorial analysis as used in the original paper was carried out. RESULTS: The Spearman-Brown correlation value was 0.8, indicating high internal reliability. The Cronbach's alpha found was 0.9, indicating a high level of internal consistency. Factorial analysis showed that four items had low factorial load and no discriminatory power, and another five items were relocated to other factors. When the instrument was applied, it showed variability to that of the original instrument. CONCLUSION: The Brazilian version of the Body Image Relationship Scale (BIRS), named Escala de Relacionamento e Imagem Corporal (ERIC), showed evidence of adequate reliability and internal consistency, making this instrument suitable to be recommended for application to Brazilian women with breast cancer, despite some limitations.
Departamento de Medicina Social da Faculdade de Medicina de Ribeirão Preto da Universidade de São Paulo - USP - Ribeirão Preto (SP), Brasil.

Departamento de Medicina Social, Faculdade de Medicina de Ribeirão Preto, Universidade de São Paulo - USP - Ribeirão Preto (SP), Brasil. 2Departamento de Psicologia e Educação, Faculdade de Filosofia Ciências e Letras de Ribeirão Preto, Universidade de São Paulo - USP Ribeirão Preto (SP), Brasil.

${ }^{3}$ Departamento de Estatística, Universidade Federal de São Carlos - UFSCar - São Carlos (SP), Brasil.

4Programa de Pós-graduação em Saúde na Comunidade, Faculdade de Medicina de Ribeirão Preto, Universidade de São Paulo - USP Ribeirão Preto (SP), Brasil.

${ }^{5}$ Departamento de Matemática Aplicada e Estatística, Instituto de Ciência Matemáticas e Computação, Universidade de São Paulo - USP São Carlos (SP), Brasil.

Conflito de interesses: nao há 


\section{Introdução}

Desde o início do século XX, teóricos das áreas de psicologia, medicina e filosofia têm se interessado pelo estudo da imagem corporal. A imagem corporal relaciona aspectos físicos, como as sensações corporais intrínsecas e extrínsecas, bem como a visão que temos de partes da superfície de nosso corpo e as impressões táteis, térmicas e de dor, que propiciam a percepção da unicidade do corpo, constituindo, assim, o esquema corporal, ou seja, a imagem tridimensional que temos de nós mesmos. A imagem corporal também envolve as representações mentais que a pessoa elabora dos outros e de si mesma, e as emoções e ações sobre a experiência do próprio corpo. Desse modo, é uma construção dinâmica e cambiável durante o ciclo vital ${ }^{1}$.

A experiência corporal é um construto fundamental para o entendimento do funcionamento humano, uma vez que a imagem corporal afeta as pessoas, desde a infância, em suas emoções, pensamentos, comportamentos da vida cotidiana e relações interpessoais, influenciando seu bem-estar subjetivo. Quando o indivíduo é desafiado por uma doença grave e potencialmente fatal como o câncer, é esperado que esse evento crítico exerça considerável impacto sobre sua imagem corporal ${ }^{2}$.

O câncer de mama e seus tratamentos podem interferir negativamente na imagem corporal da mulher, uma vez que acarretam graves consequências físicas e repercussões psicossociais que podem ser temporárias ou permanentes ${ }^{3}$. A cirurgia mamária pode ser uma experiência traumática, mesmo quando o procedimento cirúrgico altera minimamente a aparência da mama ${ }^{4,5}$.

Os tratamentos (neo)adjuvantes de quimioterapia, radioterapia e hormonioterapia desencadeiam efeitos colaterais devastadores, gerando desconfortos e alterações físicas importantes como alopecia, escurecimento ou perda das unhas, palidez, náusea, marcas ou retração da pele, ganho ou perda de peso corporal ${ }^{5,6}$.

Estudos mostram que o acometimento pelo câncer de mama resulta em prejuízos do funcionamento psicossocial, com perdas econômicas e preocupação com o comprometimento do potencial de trabalho, mudanças no relacionamento com o parceiro afetivo-sexual e com familiares e amigos. Ademais, há confrontação de preconceitos, estigmas e mudanças na vivência da sexualidade e na vida sexual, além da imagem corporal, desencadeando muitas vezes quadros de ansiedade e depressão $0^{7-10}$.

A remoção de uma das mamas muitas vezes significa, no ideário social, a perda da feminilidade, já que na história da sociedade ocidental as mamas são consideradas símbolo da identidade feminina, relacionadas à sexualidade e sensualidade e representadas como objeto de desejo e prazer. Também são símbolos da maternidade, compondo um ideal de feminilidade e atratividade ${ }^{9}$.

No Brasil observa-se intenso estímulo social para as mulheres obterem um corpo belo e sexualmente atraente, segundo um modelo social de magreza e juventude, por meio da adoção de exercícios físicos, dietas alimentares, tratamentos estéticos e cirurgias plásticas ${ }^{11}$. Dessa forma, as mulheres acometidas pelo câncer de mama podem ser especialmente afetadas quando, durante ou após os tratamentos, sua aparência física se distancia dos ideais sociais.

A avaliação da imagem corporal de mulheres com câncer de mama é importante para melhor compreender a percepção dessas mulheres acerca de si mesmas e para fomentar práticas mais eficazes de intervenção que melhorem a qualidade de vida dessas mulheres, como grupos de apoio, por exemplo.

Vários instrumentos são descritos na literatura para avaliar a imagem corporal de pacientes oncológicos ${ }^{12-14}$, alguns especificamente para o uso em câncer de mama ${ }^{13-15}$, como Body Image Scale (BIS), Body Image Questionnaire (BIQ), o Body Image after Breast Cancer Questionnaire (BIBCQ), Sexual Adjustment and Body Image Scale (SABIS), Body Image Index (BII) e Body Image and Relationship Scale (BIRS). Dessas seis escalas, quatro foram desenvolvidas para avaliar a imagem corporal de mulheres acometidas pelo câncer de mama (BII, SABIS, BIBCQ e BIRS).

O instrumento BII contempla apenas a dimensão da imagem corporal e a escala SABIS avalia apenas dois aspectos diferentes em suas subescalas (imagem corporal e ajustamento sexual). Já o BIBCQ é um instrumento, de origem canadense, que abrange cinco dimensões com domínio da imagem corporal, que são: vulnerabilidade, estigma corporal, limitações, preocupação com o corpo e preocupação com o braço. Embora bastante completa, a versão final desse instrumento apresenta 53 itens, o que pode ser limitante visto que uma escala com muitos itens pode fazer com que sua aplicação seja cansativa para as respondentes, especialmente aquelas que se encontram debilitadas em decorrência da terapêutica instituída.

Neste contexto optamos pela utilização do BIRS, elaborado nos Estados Unidos para avaliar atitudes sobre aparência, saúde, força física, sexualidade, relacionamentos e funcionamento social de mulheres submetidas aos tratamentos do câncer de mama ${ }^{14}$. Os itens incluídos na escala foram obtidos por meio de grupos focais realizados com mulheres que tiveram câncer de mama. A vantagem desse instrumento sobre os demais é sua grande abrangência de domínios e sua praticidade de aplicação. Ele foi traduzido para a língua portuguesa com o nome de Escala de Relacionamento e Imagem Corporal (ERIC) e passou por vários passos 
preconizados para sua adaptação transcultural para ser aplicado no Brasil ${ }^{16}$.

Embora a imagem corporal seja importante tema de estudo, no Brasil recente revisão da literatura ${ }^{17}$ evidenciou que há numerosos estudos publicados que utilizaram instrumentos não validados. Daí a importância desta validação.

Considerando o exposto, este estudo teve por objetivo a validação do instrumento BIRS adaptado para mulheres brasileiras acometidas pelo câncer de mama.

\section{Métodos}

Participaram do estudo mulheres usuárias do Sistema Único de Saúde (SUS) submetidas aos tratamentos do câncer de mama em um hospital público universitário entre 2006 e 2010 , originadas de um projeto de pesquisa que visou avaliar a função sexual e a imagem corporal. Por isso, o tamanho amostral foi calculado baseado na prevalência de $50 \%$ em relação à atividade sexual no último mês, considerando população finita, erro relativo de $5 \%$ e nível de significância de $\alpha=5 \%$, o que resultou em um mínimo de 132 mulheres. $\mathrm{Na}$ validação do instrumento original BIRS foi estimado um tamanho amostral de 85 participantes para o número de variáveis e fatores estabelecidos ${ }^{14}$.

As respondentes foram sorteadas de uma lista fornecida por um serviço específico de reabilitação psicossocial de mulheres em tratamento do câncer de mama e foram entrevistadas em seus domicílios, após serem contatadas por telefone ou visita domiciliar para marcar a entrevista, entre setembro de 2010 e março de 2011.

Diferentemente do instrumento original, desenhado para ser auto-aplicado, a adaptação foi realizada de modo que pudesse ser aplicado em situação face a face por entrevistadora treinada, considerando as dificuldades de entendimento que poderiam haver entre as respondentes brasileiras devido à baixa escolaridade.

A BIRS é composta por 32 assertivas, subdivididas em 3 fatores ou domínios: Força e Saúde; Barreiras Sociais; Aparência e Sexualidade. O fator "Força e Saúde" compreende 12 frases que abordam: energia para realizar atribuições físicas, força física, corpo saudável e forma física. Em relação ao fator "Barreiras Sociais", 9 assertivas abordam a restrição das atividades sociais em decorrência dos sintomas desencadeados pelo tratamento, como ondas de calor, restrição das atividades sociais em decorrência de mudanças na aparência física, restrição das atividades sociais pelo desconforto físico e vergonha ocasionada pelo tratamento de câncer de mama. O fator "Aparência e Sexualidade" é composto por 11 frases, que compreendem: aparência corporal e integridade do corpo, sexualidade e desejo sexual, constrangimento e vergonha com a aparência corporal ${ }^{14}$. As categorias de respostas utilizadas são cinco, que variam de "concordo plenamente" a "discordo plenamente", passando pela "concordância/discordância simples" ou pela resposta "nem concordo nem discordo".

\section{Procedimentos}

\section{Coleta de dados}

As entrevistas ocorreram durante todos os dias da semana, inclusive aos finais de semana e feriados, nos períodos da manhã, tarde e noite, dependendo da disponibilidade da entrevistada. Quando não era obtido o contato telefônico para marcar entrevista, as entrevistadoras fizeram até três visitas ao endereço indicado e, quando não encontrada, a mulher sorteada era excluída do estudo.

\section{Análise estatística}

Parte das assertivas da BIRS apresenta uma expressão positiva da imagem corporal, enquanto que outra parte tem sentido negativo. Para obtenção do resultado geral da escala, os itens com expressão positiva da imagem corporal tiveram sua pontuação invertida. O prejuízo ou declínio da imagem corporal é caracterizado pelo maior valor obtido a partir da somatória de todos os itens. Para avaliar a confiabilidade desse instrumento, a consistência interna foi avaliada por meio do coeficiente alfa de Cronbach total e parcial, também o coeficiente de Sperman-Brown foi obtido, utilizando Split-half, no qual o instrumento foi dividido em duas partes. Para validação do instrumento, repetimos a análise fatorial utilizada no artigo original, com objetivo de comparar os resultados. Portanto, 32 itens da escala foram submetidos a análise fatorial considerando o método "principal axis factoring" e rotação Varimax ${ }^{18,19}$. A partir dos resultados da análise fatorial fizemos modificações no instrumento e analisamos a sua aplicação em nossa amostra de mulheres.

Os dados foram processados com auxílio dos softwares STATA 9.0, que foi utilizado para estimar a frequência simples das variáveis, e SAS versão 9.0 para a análise fatorial.

O estudo foi aprovado pelo Comitê de Ética em Pesquisa da Escola de Enfermagem de Ribeirão Preto da Universidade de São Paulo (CEP/EERP-USP), processo $\mathrm{n}^{\circ} 1074 / 2009$, respeitando as diretrizes definidas pela Resolução n 466/2012 do Conselho Nacional de Saúde ${ }^{20}$.

\section{Resultados}

Foram sorteadas 184 mulheres; dessas, 14 eram falecidas, 17 se recusaram a colaborar com a pesquisa e 14 não foram localizadas, resultando em um total de 139 mulheres incluídas no estudo. 
Características sociodemográficas e clínicas da amostra

A mulher mais jovem tinha 24 anos e a mais velha 78 anos, com média de idade de 54,6 anos e mediana de 54. A maioria das entrevistadas se autoclassificou como branca ou parda, estava casada ou coabitava com um companheiro. Mais da metade delas tinha pelo menos o ensino fundamental com tempo médio de escolaridade igual a 7,3 anos. A maioria trabalhava em profissões que exigem nível médio, como vendedora, auxiliar administrativo ou professora (Tabela 1). Para evitar um possível viés de memória por parte das respondentes foi incluído no estudo, como variável de controle, o tempo de diagnóstico.

\section{Da consistência interna}

O valor de correlação Spearman-Brown obtido foi de 0,82 , o alfa de Cronbach total encontrado foi de 0,92, ambos bastante próximos de 1, indicando alta consistência interna. Os valores do alfa de Cronbach, caso alguma das questões fossem retiradas, variou de 0,92 a 0,93 , ou seja, a retirada de nenhuma das questões aumentou consideravelmente tal valor.

\section{Da análise fatorial}

A análise fatorial exploratória, como no estudo original, foi utilizada para identificar fatores de cada um

Tabela 1. Aspectos sociodemográficos das mulheres com diagnóstico de câncer de mama

\begin{tabular}{|c|c|c|}
\hline Aspectos sociodemográficos & n & $\%$ \\
\hline \multicolumn{3}{|l|}{ Idade } \\
\hline 24 a 40 anos & 14 & 10,7 \\
\hline 41 a 50 anos & 35 & 21,2 \\
\hline 51 a 60 anos & 47 & 33,8 \\
\hline Acima dos 60 anos & 43 & 31,0 \\
\hline \multicolumn{3}{|l|}{ Raça/cor } \\
\hline Branca & 88 & 63,3 \\
\hline Parda & 39 & 29,1 \\
\hline Preta & 10 & 7,2 \\
\hline Oriental & 1 & 0,7 \\
\hline Indígena & 1 & 0,7 \\
\hline \multicolumn{3}{|l|}{ Estado marital } \\
\hline Casada & 61 & 43,8 \\
\hline Coabitavam & 16 & 11,5 \\
\hline Solteira/Viúva/Separada & 62 & 45,0 \\
\hline \multicolumn{3}{|l|}{ Escolaridade } \\
\hline Menos de 4 anos & 28 & 20,1 \\
\hline $4^{\mathrm{a}}$ série fundamental & 41 & 29,5 \\
\hline $8^{a}$ série fundamental & 21 & 15,1 \\
\hline Ensino Médio & 31 & 22,3 \\
\hline \multicolumn{3}{|l|}{ Ocupação } \\
\hline Trabalha fora & 52 & 37,4 \\
\hline Dona de casa & 47 & 33,8 \\
\hline Aposentada & 33 & 23,7 \\
\hline Doméstica & 7 & 5,0 \\
\hline
\end{tabular}

dos domínios do instrumento, isto é, agrupar o total de questões presentes no instrumento em um número pequeno de domínios (subconjuntos de questões). Obteve-se a matriz de correlação entre as 32 questões (32 linhas e 32 colunas) e o resultado das correlações obtidas mostra que $50 \%$ delas são maiores do que 0,24 , ou seja, há indícios de altas correlações entre os itens do instrumento.

A medida de adequação da amostra foi satisfatória e o modelo fatorial foi adequado, o coeficiente Kaiser-MayerOlkin foi estimado (KMO=0,83) e o teste de esfericidade de Bartlett, para medir a qualidade das correlações entre as variáveis, foi estatisticamente significativo.

Apesar do critério de normatização de Kaiser, que se baseia em manter fatores com autovalores maiores que um, revelar que sete primeiros autovalores deveriam ser mantidos e poderiam gerar sete fatores, com o total de variabilidade explicada por estes fatores de $68,9 \%$, optamos por utilizar os três fatores/domínios tal como o instrumento original, o que tende a facilitar a interpretação e não é prejudicial à variabilidade explicada, agora de $51,1 \%$. Essa variabilidade se distribui em $30,8 \%$ para o fator $1,12,6 \%$ para o fator 2 e $7,7 \%$ para o fator 3 . Essa opção nos permite a comparação com o estudo original, que obteve as variabilidades 35,$3 ; 9,1$ e $8,4 \%$ para cada fator, respectivamente. Portanto, foram mantidos os fatores: Força e Saúde (fator 1), Barreiras Sociais (fator 2) e Aparência e Sexualidade (fator 3), tal como no estudo americano original ${ }^{14}$, por sua variabilidade assemelhar-se muito. Com o objetivo de facilitar a interpretação das cargas fatoriais (fatores independentes), foi considerado rotação Varimax, resultado cujas cargas fatoriais são apresentadas em material suplementar: http://febrasgo. org.br/rbgo/SM/5354-10/Quadro\%201.pdf

Quatro questões (12, 22, 28 e 32) com carga fatorial abaixo de 0,4 não foram classificadas em nenhum dos fatores, já que suas cargas fatoriais foram similares tanto no fator 2 quanto no 3, portanto, identificadas como sem poder discriminatório, foram excluídas da análise final que determina a pontuação do instrumento.

Os resultados da análise fatorial segundo maior carga fatorial obtida e fator referente às maiores cargas fatoriais obtidas para cada questão foram obtidas tanto para o instrumento original (BIRS) ${ }^{14}$ quanto para o presente estudo (ERIC). Além das questões citadas com carga fatorial baixa, observamos que houve mudança de domínio de cinco questões como o caso da 31, "Sentia que tinha algum controle sobre a minha (condição de) saúde", que na análise fatorial foi relacionada ao fator 1 e não ao 3 , como era no estudo original ${ }^{14}$. $\mathrm{O}$ mesmo aconteceu com as questões 3 e 11, "Minha falta de energia me fazia sentir constrangida" e "Eu me sentia constrangida e envergonhada porque estava fora de forma”, que apresentaram maior carga no segundo (Barreiras Sociais) e não no 
primeiro fator (Força e Saúde). A questão 16, "Mudanças na minha aparência física, que atribuo a minha cirurgia de câncer de mama, não me deixavam fazer coisas que eu queria fazer", apresentou maior carga no fator 3 e não no fator 2. A questão 32 "Atividade sexual era uma parte importante da minha vida" apresentou carga fatorial igual a 0,26, próxima daquela obtida no BIRS, entretanto, a classificação no fator foi diferente, classificou-se no fator 1 (Força e Saúde) enquanto no BIRS estava no fator 3 (Aparência e Sexualidade). Informações detalhadas sobre os resultados obtidos com os instrumentos BIRS e ERIC podem ser consultados em: http://febrasgo.org.br/rbgo/ SM/5354-10/Quadro\%202.pdf

\section{Das pontuações obtidas pelo instrumento}

Escala de Relacionamento e Imagem Corporal

Baseado nesses resultados obtidos para o instrumento ERIC, modificamos o instrumento, excluindo as quatro perguntas e realocando as outras cinco em outros domínios. Assim, calculamos a pontuação de cada uma das mulheres nos três fatores e também uma pontuação geral. Os componentes identificados foram denominados como fator 1 'Força e Saúde' (10 itens), fator 2 'Barreiras sociais' (10 itens) e fator 3 'Aparência e Sexualidade' (8 itens).

A classificação utilizada para os fatores foi baseada em pontos de cortes considerando a distribuição dos pontos obtidos pelos quartis (25; 50 e 75\%), já a classificação geral foi obtida considerando os quartis superiores ( 25 e $75 \%$ ).

A pontuação para percepção geral da imagem corporal apresentou-se alta para valores menores que 67 , média para valores entre 67 e 98 e baixa para valores maiores que 98.

Aplicada nas 139 participantes, a classificação geral obteve uma média de 82,4 pontos, com desvio padrão (DP) de 18,9; escore mínimo de 45 e máximo de 125. Metade das participantes, 70 (50,4\%), obtiveram um escore médio, o que sugere que a imagem corporal não estava prejudicada; 38 delas $(27,2 \%)$ apresentaram valores baixos de pontuação, sugerindo uma imagem corporal positiva, enquanto que 31 mulheres $(22,3 \%)$ obtiveram escores compatíveis com imagem corporal prejudicada.

Em relação aos fatores, aproximadamente metade das mulheres apresentaram classificação média nos três domínios da escala. O fator 1 (Força e Saúde), com 10 itens, foi definido como percepção alta para valores menores de 25 , média alta para valores entre 25 e 35 , média baixa entre 35 e 40 e baixa para maior que 40. Em nossa amostra, a pontuação variou de 10 a 50 e a média obtida foi de 31,9 pontos.

Para o fator 2 (Barreiras Sociais), que também contempla 10 questões, definiu-se: percepção alta para valores menores que 21; média alta para valores entre 21 e 26; média baixa para valores entre 26 e 34 e baixa para valores maiores que 34 pontos. As mulheres do estudo apresentaram pontuação que variou de 11 a 49 e a pontuação média de 27,4 pontos.

Para Aparência e Sexualidade, fator 3, com 8 itens, definiu-se: percepção alta valores menores que 17; média alta para valores entre 17 e 24; média baixa para valores entre 24 e 30 e baixa para valores maiores que 30 pontos. Os resultados da pontuação variaram de 8 a 40, a média encontrada foi de 23,2 pontos.

O tempo de diagnóstico foi categorizado em menor igual a 2 anos e maior que 2 anos e apresentou associação estatística significante com a pontuação da ERIC (Tabela 2). A percepção negativa da imagem corporal foi maior no grupo com maior tempo de diagnóstico.

Tabela 2. Percepção da imagem corporal (ERIC geral versus tempo de diagnóstico)

\begin{tabular}{lcc}
\hline Percepção ERIC geral & Menor ou igual a 2 anos & Maior do que 2 anos \\
\hline Alta & 24 & 11 \\
Media & 20 & 40 \\
Baixa & 14 & 19 \\
Total & 58 & 70 \\
\hline
\end{tabular}

ERIC: Escala de Relacionamento e Imagem Corporal; $\chi^{2}=11,23 ; p=0,004$; valores perdidos $=11$

\section{Discussão}

A BIRS já havia sido utilizada e avaliada em outros estudos. Em um estudo, realizado pelos autores da referida escala, foi avaliado o impacto de um programa de intervenção de exercícios físicos na imagem corporal de mulheres com linfedema. O uso da BIRS permitiu que os autores concluíssem que o programa de exercícios físicos teve efeitos benéficos na autopercepção da aparência, saúde, força física, sexualidade, relacionamentos e funcionamento social das mulheres independentemente de ter o linfedema. E destacaram que a BIRS contempla aspectos da imagem corporal que outras escalas de qualidade de vida não alcançam ${ }^{21}$.

A escala também já foi utilizada em uma pesquisa coreana que avaliou fatores psicossociais (imagem corporal, sexualidade e relacionamentos sociais) relacionados ao câncer de mama e fatores genéticos que influenciam o desenvolvimento de sintomas de depressão em mulheres ${ }^{22}$.

Duas revisões sistemáticas da literatura consideraram, em seus achados, que no Brasil há uma lacuna de instrumentos desenvolvidos ou culturalmente adaptados para avaliação da imagem corporal ${ }^{17,23}$. Além disso, um desses estudos mostrou que não existem escalas criadas ou adaptadas para avaliar a imagem corporal de mulheres acometidas pelo câncer de mama ${ }^{23}$.

Em sua validação no estudo original, a escala foi aplicada, inicialmente, em 95 mulheres com idade entre 37 e 77 anos, com sobrevida ao diagnóstico de câncer de 5 a 15 anos $^{14}$. Comparando-se os resultados dos testes estatísticos 
utilizados na validação da escala norte-americana aos resultados obtidos no Brasil, há evidências de que a ERIC apresenta semelhantes propriedades, particularmente em relação à consistência interna e validação do instrumento.

O tempo de diagnóstico mostrou associação com a percepção geral, o que poderia nos levar a pensar em um viés de memória, no entanto, observa-se que a percepção negativa concentra-se no grupo com mais tempo, e não no grupo com menor tempo de diagnóstico.

Por fim, a partir da análise descritiva das pontuações foi possível tomar decisões em relação à imagem corporal das mulheres de acordo com sua pontuação em cada um dos fatores. Assim, decidiu-se por classificá-las de acordo com a pontuação recebida.

Baseado na hipótese de que alguns itens devam ser retirados, em especial as questões 12, 2228 e 32, e outros realocados, procedemos assim na aplicação da escala. Há, entretanto, a possibilidade de explicação no significado dessas questões que podem estar relacionados ao modo como a sexualidade feminina é tratada na sociedade brasileira em contraste com a sociedade norte-americana, o que poderia ser um dos principais limites desse instrumento. Entretanto, acreditamos que esse instrumento possa ser utilizado no Brasil, desde que seus limites sejam compreendidos. Esses poderiam ser superados se novas pesquisas fossem conduzidas, seja com população maior ou com redução do número de itens, e até mesmo estudando item a item ou readaptando algumas questões, em especial o fator 3 , à realidade brasileira.

Em comparação com outras escalas de imagem corporal utilizadas em pacientes oncológicos, como BIBCQ, SABIS, BII, BIQ e BIS, observa-se que apenas BIS, BIRS e SABIS apresentam o teste-reteste de confiabilidade; e somente BIRS e BIS apresentam os procedimentos de análise fatorial que permitiram a formulação definitiva dos instrumentos ${ }^{15}$. Neste artigo não foram encontrados dados sobre validação transcultural dessas escalas.

Após a adaptação da BIRS para a cultura brasileira (a ERIC) e do teste desse instrumento em 139 mulheres diagnosticadas com câncer de mama, concluímos que a ERIC pode ser uma opção para avaliação da imagem corporal de brasileiras acometidas pelo câncer de mama, confiável, apesar dos limites. Nesse sentido, o presente estudo traz como contribuição a disponibilização da ERIC para ser utilizada na prática profissional ou em pesquisas.

Apesar da diferença em relação à aplicação do instrumento original (no Brasil, na forma de entrevistas face a face, para que seu uso pudesse ser ampliado às participantes com baixa escolaridade) e sua tradução e adaptação para a língua portuguesa, o instrumento, pelos testes realizados, apresenta, em geral, validade similar à versão norte-americana.

O seu uso em futuras pesquisas permitirá ampliar a compreensão acerca das repercussões dos tratamentos do câncer de mama para a imagem corporal das mulheres brasileiras, possibilitando avaliar o foco de preocupação da mulher nas questões da saúde e força física, barreiras sociais ou aparência e sexualidade.

\section{Agradecimentos}

À Fundação de Amparo à Pesquisa do Estado de São Paulo (FAPESP) pelo suporte ao projeto "Sexualidade e câncer de mama" (FAPESP, processo n 2009/50319-8).

\section{Referências}

1. Schilder P. A imagem do corpo: as energias construtivas da psique. 3a ed. São Paulo: Martins Fontes; 1999.

2. Pruzinsky T, Cash TF. Understanding body images: historical and contemporary perspectives. In: Cash TF, Pruzinsky T, editors. Body image: a handbook of theory, research, and clinical practice. New York: Guilford; 2002. p. 3-12.

3. Silva G, Santos MA. Stressors in breast cancer post-treatment: a qualitative approach. Rev Latino-Am Enfermagem. 2010;18(4):688-95.

4. Ussher JM, Perz J, Gilbert E. Changes to sexual well-being and intimacy after breast cancer. Cancer Nurs. 2012;35(6):456-65.

5. White CA. Body images in oncology. In: Cash TF, Pruzinsky T, editors. Body image: a handbook of theory, research, and clinical practice. New York: Guilford; 2002. p. 379-86.

6. Ussher JM, Perz J, Gilbert E. Information needs associated with changes to sexual well-being after breast cancer. J Adv Nurs. 2013;69(2):327-37.
7. Santos DB, Ford NJ, Santos MA, Vieira EM. Breast cancer and sexuality: the impacts of breast cancer treatment on the sex lives of women in Brazil. Cult Health Sex. 2014;16(3):246-57.

8. Scorsolini-Comin F, Santos MA, Souza LV. Vivências e discursos de mulheres mastectomizadas: negociações e desafios do câncer de mama. Estud Psicol (Natal). 2009;14(1):41-50.

9. Santos DB, Vieira EM. Les scripts de la sexualité chez les femmes brésiliennes vivant avec un cancer du sein. Sexologies. 2014;23(1):3-7.

10. Santos DB, Vieira EM. Imagem corporal de mulheres com câncer de mama: uma revisão sistemática da literatura. Ciênc Saúde Coletiva. $2011 ; 16(5): 2511-22$.

11. Goldenberg M. Gênero, "o corpo" e "imitação prestigiosa" na cultura brasileira. Saúde Soc. 2011 ; 20(3):543-53.

12. Hopwood P, Fletcher I, Lee A, Al Ghazal S. A body image scale for use with cancer patients. Eur J Cancer. 2001;37(2): 189-97. 
13. Baxter NN, Goodwin PJ, McLeod RS, Dion R, Devins G, Bombardier C. Reliability and validity of the body image after breast cancer questionnaire. Breast J. 2006;12(3):221-32.

14. Hormes JM, Lytle LA, Gross CR, Ahmed RL, Troxel AB, Schmitz $\mathrm{KH}$. The body image and relationships scale: development and validation of a measure of body image in female breast cancer survivors. J Clin Oncol. 2008;26(8):1269-74.

15. Annunziata MA, Giovannini L, Muzzatti B. Assessing the body image: relevance, application and instruments for oncological settings. Support Care Cancer. 2012;20(5):901-7.

16. Borsa JC, Damásio BF, Bandeira DR. Adaptação e validação de instrumentos psicológicos entre culturas: algumas considerações. Paidéia (Ribeirão Preto). 2012;22(53):423-32.

17. Laus MF, Kakeshita IS, Costa TM, Ferreira ME, Fortes LS, Almeida SS. Body image in Brazil: recent advances in the state of knowledge and methodological issues. Rev Saúde Pública. 2014;48(2):331-46.

18. Hair JF Jr, Anderson RE, Tatham RL, Black WC. Análise multivariada de dados. 5a ed. Porto Alegre: Bookman; 2005.
19. Johnson RA, Wichern DW. Applied multivariate statistical analysis. 5th ed. Upper Saddle River: Prentice Hall; 2002.

20. Brasil. Ministério da Saúde. Resolução n ${ }^{\circ} 466$, de 12 de dezembro de 2012. Dispõe sobre as diretrizes e normas regulamentadoras de pesquisas envolvendo seres humanos. Brasília: Conselho Nacional de Saúde; 2012.

21. Speck RM, Gross CR, Hormes JM, Ahmed RL, Lytle LA, Hwang WT, et al. Changes in the Body Image and Relationship Scale following a one-year strength training trial for breast cancer survivors with or at risk for lymphedema. Breast Cancer Res Treat. 2010;121(2):421-30.

22. Kim KR, Chung HC, Lee E, Kim SJ, Namkoong K. Body image, sexual function and depression in Korean patients with breast cancer: modification by 5-HTT polymorphism. Support Care Cancer. 2012;20(9):2177-82.

23. Gonçalves CO, Tavares MC, Campana AN, Cabello C, Shimo AKK. Instrumentos para avaliar a imagem corporal de mulheres com câncer de mama. Psicol Teor Prat. 2012; $14(2): 43-55$. 